\title{
Propulsion of Ripples on Glass by Ion Bombardment
}

\author{
P.F. A. Alkemade \\ National Center of High Resolution Electron Microscopy and Kavli Institute of Nanoscience, Delft University of Technology, \\ Lorentzweg 1, 2628 CJ Delft, The Netherlands \\ (Received 25 August 2005; published 17 March 2006)
}

\begin{abstract}
The propulsion of surface ripples on $\mathrm{SiO}_{2}$ by an ion beam was investigated by in situ electron microscopy. The observed propagation of the ripples contradicts existing models for ion-beam-induced ripple development. A new model based on the Navier-Stokes relations for viscous flow in a thin layer is introduced. It includes inhomogeneous viscous flow, driven by spatial variations in the deposition of the energy of the ion beam. The model explains the observed reversed propagation. The hitherto unknown propulsion mechanism is important for understanding nanoscale pattern formation by ion bombardment.
\end{abstract}

Modification of surfaces is a widely explored and technologically highly relevant field of research. Surfaces bombarded by a beam of ions erode, but they may also develop specific nanoscale geometrical patterns [1]. These patterns have been studied intensively because they reveal details in the interaction of energetic particles with matter and because they have implications for ion-beam sputter erosion and deposition [2]. Recently, the formation of surface patterns by ion bombardment received appreciation as templates for the growth of low-dimensional nanostructures [3]. Various theories describe aspects of beaminduced pattern formation. Although they are widely applied, not all relevant processes are understood, not even qualitatively.

A very common pattern is a series of ripples that can form under oblique incidence of kilo-electron-volt ions. Sigmund showed that the dependence of the material's erosion rate on the curvature of the surface leads to the growth of surface irregularities [4]. Bradley and Harper (BH) used Sigmund's model to explain the appearance of ripple patterns with a distinct wavelength [5]: Ripple formation is the result of a competition between curvaturedependent roughening and smoothing by thermal surface diffusion. The original $\mathrm{BH}$ model has been extended to include smoothening by beam-enhanced surface diffusion [6] and beam-enhanced viscous flow [7]. Carter suggested that viscous relaxation of a thin surface layer, compressively stressed by the ion bombardment, causes ripple formation [8], and Rudy and Smirnov used the NavierStokes relations to describe ion-beam-enhanced viscous flow [9]. Recently, Umbach, Headrick, and Chang showed that the enhanced viscous flow is limited to the ion penetration layer [10]. A common factor of all models is that only the ripples' growth rate has been tested experimentally. In contrast, the notion that ripples propagate across the surface is rarely outspoken $[5,11]$ but always implicitly assumed. The overall surface erosion velocity is $-\nu_{0}(\theta)=$ $-Y(\theta) f \cos \theta / n$. Here $Y(\theta)$ is the number of removed atoms per incident ion, $\theta$ the angle between the ion beam and the surface normal, $f$ the ion flux, and $n$ the atomic density of the solid; the minus sign reflects the recession of the surface. Because of the increase of $Y$ with $\theta$ up to $\sim 75^{\circ}$, the slopes that face the incident ion beam (the up slopes; see the inset in Fig. 2) erode slower than the opposite (viz., the down) slopes, except for grazing incidence. Hence, ripples should move against the incident beam direction. Their velocity is [5]

$$
\begin{aligned}
u & =-\frac{d \nu_{0}(\theta)}{d \theta}=-\frac{f}{n}\left(\cos \theta \frac{d Y}{d \theta}-Y \sin \theta\right) \\
& =-\nu_{0}\left(\frac{1}{Y} \frac{d Y}{d \theta}-\tan \theta\right) \equiv u_{1},
\end{aligned}
$$

which is negative up to $\theta \sim 65^{\circ}$. Despite the numerous studies on ripple growth, there are only a very few studies on ripple propagation [12-15]. Nevertheless, ripple propagation is a phenomenon that can be as important for our understanding of pattern formation as ripple growth. Experimental studies on ripple development are almost exclusively based upon ex situ or postmortem spatially resolved techniques, e.g., via surface imaging with atomic force microscopy (AFM) or scanning electron microscopy (SEM). Although individual ripples are observed, only the final state of a bombarded surface area is being recorded. On the other hand, in-situ real-time studies are based upon changes in the reflection of a broad beam from an ensemble of ripples $[7,16]$ and, therefore, do not expose the fate of individual ripples. Recently, Datta, Wu, and Wang used focused ion-beam microscopy to induce and observe ripple formation on diamond but could not detect any movement [13]. Habenicht et al. observed moving ripples on Si [14], but the bombarded area was so small that its boundaries determined ripple development [15].

In this Letter, we present the first clear observation of the propulsion of surface ripples by ion bombardment. For this study, we used a scanning electron microscope equipped with a focused ion beam (FIB). The bombarded material is $\mathrm{SiO}_{2}$; the beam is $\mathrm{Ga}^{+}$ions. Surprisingly, we do indeed observe ripple propulsion but in the direction opposite to that predicted by Eq. (1). Newly formed ripples are initially 
stationary and then drift slowly in the direction of the projected beam, contradicting all existing models. We explain this behavior by inhomogeneous enhanced viscous flow in the penetration layer of the ions. The inhomogeneities in the flow are a consequence of an inhomogeneous stress, which - in its turn - results from the differences in the ions' energy deposition between the up and down slopes of the ripples.

In our study, borosilicate glass and a $500 \mathrm{~nm} \mathrm{SiO}$ layer on Si have been investigated in a dual-beam (FIB + SEM) instrument (FEI Strata DB235). The current of the scanning $30 \mathrm{keV} \mathrm{Ga}^{+}$beam was $500 \mathrm{pA}$. The scanned area was $24 \times 24 \mu \mathrm{m}^{2}$; the mean flux $f$ was $5.4 \times 10^{18}$ ions $^{-1} \mathrm{~m}^{-2}$. The spot size was relatively large $(\sim 1 \mu \mathrm{m})$ to avoid surface structure formation related to the scanning mode of the beam. Each surface spot was bombarded during $10 \mu \mathrm{s}$; repetition time was $100 \mathrm{~ms} ; \theta$ was $45^{\circ}$. Each 135 seconds of bombardment - in which $45 \mathrm{~nm}$ of material was removed-was alternated with electron beam imaging. To avoid apparent lateral shifts of surface features with the change in height, images were taken with the surface perpendicular to the electron beam. In addition, we have measured the sputter yield $Y(\theta)$ for $0^{\circ}<\theta<67^{\circ}$.

Figure 1 shows sections of SEM images of an area at various stages of bombardment. Visible surface defects and separately milled lines outside the bombarded area serve as position markers to correct for stage drifts $(\sim 100 \mathrm{~nm})$ between consecutive SEM images. The first image (a) shows faintly a few ripples induced downstream of a presumably preexisting surface defect. Later [(b)-(d)], more ripples appear at regular time intervals further downstream, and then (d) ripples emerge everywhere. The relation between the amount of removed material and the (central) position of a number of adjacent dark lines in the SEM images is shown in Fig. 2. The lower series 1-6 emerged behind the presumed surface irregularity. The first of these induced ripples is initially stationary, and only much later it starts to move, while ripples $2-6$ propagate almost directly after their appearance. The spontaneously emerging ripples $a-g$ are initially stationary but quickly start to move. Finally, all ripples propagate with a velocity of $\sim 0.75 \nu_{0}$. The wavelength, or mean ripple-to-ripple distance, of the spontaneous ripples is $225 \mathrm{~nm}$; of the induced ones, it is $195 \mathrm{~nm}$. The line "BH model" in Fig. 2 shows the propagation according to Eq. (1). Additionally, we observed that, if $\theta<$ $35^{\circ}$, no ripples appear, and, if $\theta>55^{\circ}$, ripples emerge and grow very rapidly and transform quickly into irregular steep edges and flat plateaus. Experiments in which the beam focusing, scanning directions, and speeds were varied did not result in noticeably different patterns. Further, we observed a $25 \%$ decrease of the relative propagation speed $\left(=\left|u / \nu_{0}\right|\right)$ with a tenfold increase of the flux $f$. There was no difference between the oxidized Si sample and glass. Postmortem AFM revealed an average ripple amplitude of $\sim 5 \mathrm{~nm}$ after $600 \mathrm{~nm}$ of erosion.

Our SEM observations confirm numerous studies: Ionbeam bombardment can induce surface ripples. However,
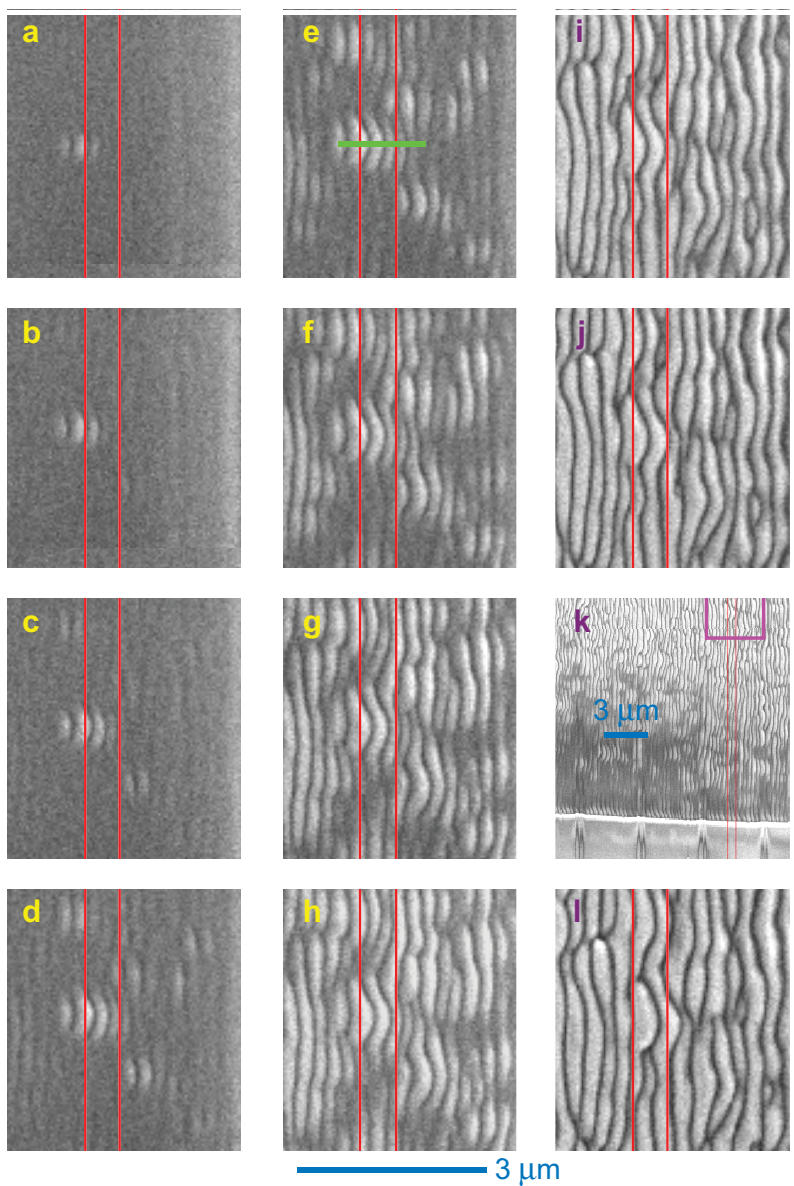

FIG. 1 (color). Sections of SEM images (top view) of a glass surface during $30 \mathrm{keV} \mathrm{Ga}$ bombardment; fluence in (a) is $21 \times$ $10^{20}$ ions $/ \mathrm{m}^{2}$, increasing by $5.2 \times 10^{20}$ ions $/ \mathrm{m}^{2}$ per image; the beam entered from the left; the vertical lines are reference lines. In (a) a single ripple emerges and in (d) a complete ripple pattern. There is a steady drift of the ripples to the right. The positions of a few ripples - the green line in (e) - are plotted in Fig. 2 (1-6). Image $k$ shows $25 \%$ of the entire crater $(20 \%$ of the SEM image) with position marker lines outside the crater.

the observed propagation of the ripples contradicts all existing models: It is slower, not constant, and in the opposite direction. Because it is unlikely that Eq. (1) does not apply here, one more mechanism must be at play. We hypothesize here that local variations in energy deposition lead to gradients in viscous flow, which in their turn propel the ripples.

We put forward a new model that includes the NavierStokes relations for viscous flow in a thin layer. The flow is enhanced by thermal spikes that are generated by the incident ions. We note that a series of studies shows the important role of thermal spikes in shape changes of amorphous objects bombarded by energetic ions $[17,18]$. The inset in Fig. 2 depicts a cross section of a rippled surface with a surface height of $H(x)=A \sin (q x)$. The ions deposit their kinetic energy $E$ along their path of penetration. Neglecting the ions' lateral spread, the lower boundary of 


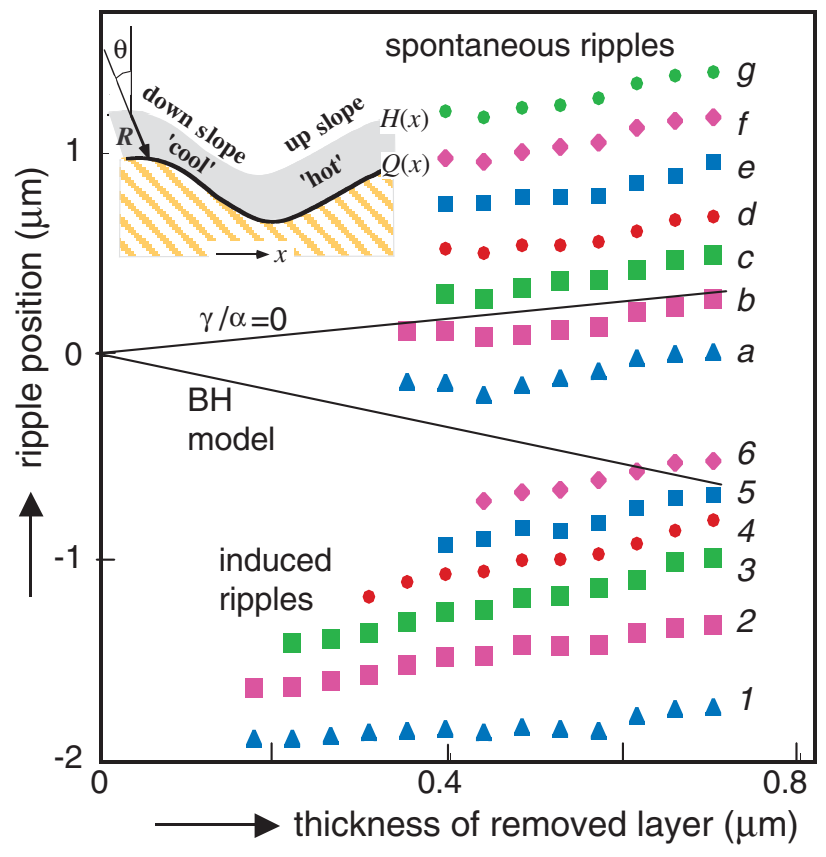

FIG. 2 (color). Ripple position versus thickness of the removed layer. The inset shows a cross section of the penetration layer. $H(x)$ is the surface and $Q(x)$ the lower boundary of the penetration layer; $R$ denotes the range of the ions. The ripples 1-6 emerged behind a presumed surface irregularity (see Fig. 1); the ripples $a-g$ emerged spontaneously. The lower line is the Bradley and Harper model [5]; the upper one (" $\gamma / \alpha=0$ ") is the new model for viscous flow driven by thermal-spike stress.

the penetration layer is a translation of the surface: $Q(x)=$ $-R_{\perp}+A \sin \left[q\left(x-R_{\|}\right)\right]$. We take the $z$ direction normal to the surface and let the ions move in the positive $x$ and negative $z$ directions. Further, $R_{\|} \equiv R \sin \theta$ and $R_{\perp} \equiv$ $R \cos \theta, R$ being the penetration range ( $25 \mathrm{~nm}$ in our study [19]). The thickness of the penetration layer is $G(x) \equiv$ $H(x)-Q(x)$ [20]. In our model, we use the concept of the thermal spike: The energy deposition of the ionsactually discrete in space and time - is effectively a temporary heating by $\Delta T_{0}$ [21]. The observed nearindependency of $\left|u / \nu_{0}\right|$ on the ion flux $f$ indicates that this concept is indeed applicable. Incidentally, the energy loss per unit of path length is almost constant [19]. Thus, the energy of the beam is deposited almost uniformly in the penetration layer, and, therefore, the total energy deposition below a surface site is proportional to the penetration layer thickness below that site. We postulate that the local heating varies proportionally to the local thickness of the penetration layer: $\Delta T(x)=\Delta T_{0} G(x) / R_{\perp}$. We assume further that heating by the ion beam leads to a stress $\sigma(x)=$ $-Y_{m} \varepsilon \Delta T(x)=-Y_{m} \varepsilon \Delta T_{0} G(x) / R_{\perp} \equiv-\alpha G(x)$ in the penetration layer $\left(Y_{m}\right.$ is the Young's modulus and $\varepsilon$ is the thermal expansion coefficient). We use the NavierStokes relations to express the change in surface height by viscous flow in a thin undulating layer with variable stress [22]. This term is added to the change in surface height according to the extended BH model [5-7]. We get

$$
\begin{aligned}
H_{t}= & -\nu_{0}-u_{1} H_{x}+\frac{\Gamma R Y f \cos \theta}{n} H_{x x}-\left(B H_{x x}\right)_{x x} \\
& +\left[\frac{G^{3}}{3 \eta}\left(-\gamma H_{x x}-\sigma\right)_{x}\right]_{x}
\end{aligned}
$$

in which the subscripts $t$ and $x$ denote differentiation to $t$ and $x$, respectively; $u_{1}$ is given by Eq. (1), $\Gamma$ is the parameter from the BH model, and $B$ is the surface diffusivity. The last (5th) term is the added one. We assume further that surface tension $\gamma$ and the beam-reduced viscosity $\eta$ are independent of temperature. Moreover, we write $\eta^{-1}$ as $f \cos \theta \eta_{r}^{-1}$, where $\eta_{r}^{-1}$ is the reciprocal viscosity per incident ion [7], and similarly $B$ as $f \cos \theta B_{r}$. It is convenient to discard the obvious general surface recession $-\nu_{0}$ and to write solutions of Eq. (2) in terms of wave functions with wave number $q$ and phase velocity $\omega$. Thus,

$$
\begin{aligned}
H^{*}(x, t) & =A \exp (r t) \cdot \exp [i(q x-\omega t)], \\
Q^{*}(x, t) & =-R_{\perp}+A \exp (r t) \cdot \exp [i(q x-\omega t)] \cdot \exp \left(-i q R_{\|}\right), \\
G^{*}(x, t) & =R_{\perp}+H^{*}(x, t) \cdot\left[1-\exp \left(-i q R_{\|}\right)\right] \\
& =R_{\perp}+\zeta H^{*}(x, t),
\end{aligned}
$$

with $\zeta \equiv 1-\exp \left(-i q R_{\|}\right)$. By inserting Eq. (3) into Eq. (2) and performing the differentiations, one gets

$$
\begin{aligned}
\frac{r-i \omega}{f \cos \theta} H^{*}= & \frac{-i u_{1} q H^{*}}{f \cos \theta}-\beta q^{2} H^{*}-B_{r} q^{4} H^{*} \\
& -\frac{\gamma q^{2}+\alpha \zeta}{3 \eta_{r}} G^{* 2} q^{2}\left(G^{*}+3 \zeta H^{*}\right) H^{*},
\end{aligned}
$$

with $\beta \equiv \Gamma R Y / n$. Using $\zeta \approx i q R_{\|}+\frac{1}{2} q^{2} R_{\|}^{2}$, taking the first order in $H^{*}$, and separating the real and imaginary parts lead to

$$
\frac{r}{f \cos \theta} \approx-\beta q^{2}-B_{r} q^{4}-\frac{\left(\gamma+\frac{1}{2} \alpha R_{\|}^{2}\right)}{3 \eta_{r}} R_{\perp}^{3} q^{4}
$$

and

$$
\frac{\omega}{f \cos \theta} \approx \frac{u_{1} q}{f \cos \theta}+\frac{\alpha R_{\|}}{3 \eta_{r}} R_{\perp}^{3} q^{3} .
$$

(Note that $G_{x}^{*}=\zeta H_{x}^{*}$.) Equation (5a) is similar to the one introduced by Umbach, Headrick, and Chang [10], apart from the $1 / 2 \alpha R_{\|}^{2}$ term. Because $u_{1}$ is negative, the wave velocity $\omega / q$ is positive or negative, depending on the stress $(v i z . \alpha)$ and the viscosity $\left(\eta_{r}\right)$. One can eliminate one parameter by using the fact that the growth rate function $r(q)$ has its maximum at the observed wave number $q_{m}$ of $28 \mu \mathrm{m}^{-1}(=2 \pi / 225 \mathrm{~nm})$ [7]. If we also set $B_{r}=0$, the radiation-reduced viscosity $\eta_{r}$ must be

$$
\eta_{r}=\frac{-4\left(\gamma+\frac{1}{2} \alpha R_{\|}^{2}\right) R_{\perp}^{3} q_{m}^{2}}{6 \beta}=\frac{\left(2 \gamma+\alpha R_{\|}^{2}\right) n R_{\perp}^{2} q_{m}^{2} \cos \theta}{-3 \Gamma Y}
$$

and the ripple velocity 


$$
\begin{aligned}
u & =\frac{\omega}{q_{m}}=u_{1}+\frac{\alpha R_{\|} R_{\perp}^{3} f \cos \theta q_{m}^{2}}{3 \eta_{r}}=u_{1}+\frac{-\Gamma Y f \cos \theta}{\left(\frac{2 \gamma}{\alpha R_{\|}^{2}}+1\right) n \sin \theta} \\
& =-\nu_{0}\left(\frac{Y_{\theta}}{Y}-\tan \theta\right)+\nu_{0} \frac{-\Gamma}{\left(\frac{2 \gamma}{\alpha R_{\|}^{2}}+1\right) \sin \theta} \equiv u_{1}+u_{2} .
\end{aligned}
$$

Note that $u$ does not depend on $q_{m}$ nor on $\eta_{r}$. From our experimental conditions and the properties of glass, we can make quantitative estimates. For $30 \mathrm{keV} \mathrm{Ga}$ on $\mathrm{SiO}_{2}$ at $\theta=$ $45^{\circ}, \Gamma=-0.95$. We have measured $Y\left(\theta=45^{\circ}\right)=5.6 \pm$ 0.5 atoms/ion and $Y^{-1} d Y / d \theta=1.9$. Hence, $u_{1}=$ $-0.90 \nu_{0}$. If $\gamma=0.3 \mathrm{~J} \mathrm{~m}^{-2}, \varepsilon=8 \times 10^{-7} \mathrm{~K}^{-1}$, and $Y_{m}=7.5 \times 10^{10} \mathrm{~Pa}[23]$, then $\eta_{r}=1.0 \times 10^{27} \mathrm{~Pa} \mathrm{~m}^{-2}$ [Eq. (6)] and $r=3.0 \times 10^{-3} \mathrm{~s}^{-1}$, corresponding to $\sim 50 \%$ growth in ripple amplitude per frame of $135 \mathrm{~s}$ in Fig. 1. From Eq. (7), we get $u_{2}=0.80 \nu_{0}$. The experimental data of Fig. 2 imply a flow-related velocity $u_{2}$ of $1.65 \nu_{0}$. Our value for $\eta_{r}$ is in good agreement with the literature: $\sim 1 \times 10^{27} \mathrm{~Pa} \mathrm{~m}^{-2}$ at an ion energy loss of $1 \mathrm{keV} \mathrm{nm}^{-1}$ [18], although for the higher beam energies in Ref. [18] the main mechanism of energy transfer is not by nuclear collisions but by electronic excitations.

The calculation results depend on the values for the surface tension, Young's modulus, etc. Their values, or their meaning, during the thermal spikes are uncertain. Furthermore, we neglected beam-enhanced surface diffusion and redeposition of sputtered atoms. Inclusion of smoothing by diffusion or uniform redeposition would imply less smoothing by viscous flow and, thus, $u_{2}=$ $<0.80 \nu_{0}$. If one also assumes that the surface tension is a negligible driving force $\left(2 \gamma \ll \alpha R_{\|}^{2}\right)$, the calculated flowrelated ripple velocity $u_{2}$ is $1.34 \nu_{0}$, slightly lower than measured $\left(1.65 \nu_{0}\right)$. Hence, despite these uncertainties, we see good agreement between theory and experiment. We note that the flow model of Rudy and Smirnov predicts no observable ripple propagation [9], supposedly because it takes the minute momentum of the ions as the only driving force.

To summarize, we have shown experimentally that ionbeam-induced surface ripples on glass propagate. The observed propagation is not in accordance with the Bradley and Harper model for ripple development nor with related models that include (beam-enhanced) surface diffusion and surface-energy-driven viscous flow. A new model, that treats viscous flow in terms of the NavierStokes relations, can explain the reversed propagation. The newly identified mechanism in ripple development is spatial variations in energy deposition: The ripples' up slopes absorb more energy of the incident ions than the down slopes. As a consequence, stress differences are generated, which enhance viscous flow from the up slopes to the down slopes. This mechanism compensates the negative propagation caused by the faster erosion of the down slopes. Knowledge of this hitherto unknown mechanism opens new routes for understanding and exploiting surface pattern formation and shape changes by ion-beam bombardment.

[1] G. Carter, J. Phys. D: Appl. Phys. 34, R1 (2001).

[2] S. M. Allameh, N. Yao, and W. O. Soboyejo, Scr. Mater. 50, 915 (2004); D. P. Adams et al., J. Vac. Sci. Technol. B 21, 2334 (2003).

[3] S. Facsko et al., Science 285, 1551 (1999); B. Kahng et al., Appl. Phys. Lett. 78, 805 (2001); M. Castro, R. Cuerno, L. Vazquez, and R. Gago, Phys. Rev. Lett. 94, 016102 (2005).

[4] P. Sigmund, J. Mater. Sci. 8, 1545 (1973).

[5] R. M. Bradley and J. M. E. Harper, J. Vac. Sci. Technol. A 6, 2390 (1988).

[6] S. W. MacLaren, J. E. Baker, N. L. Finnegan, and C. M. Loxton, J. Vac. Sci. Technol. A 10, 468 (1992).

[7] T. M. Mayer et al., J. Appl. Phys. 76, 1633 (1994); E. Chason, T. M. Mayer, B. K. Kellerman, D. T. McIlroy, and A. J. Howard, Phys. Rev. Lett. 72, 3040 (1994).

[8] G. Carter, Surf. Interface Anal. 25, 952 (1997).

[9] A.S. Rudy and V. K. Smirnov, Nucl. Instrum. Methods Phys. Res., Sect. B 159, 52 (1999).

[10] C. C. Umbach, R. L. Headrick, and K.C. Chang, Phys. Rev. Lett. 87, 246104 (2001).

[11] G. Carter, Phys. Rev. B 59, 1669 (1999); M. Makeev et al., cond-mat/0007354.

[12] I. Koponen, M. Hautala, and O.-P. Sievänen, Phys. Rev. Lett. 78, 2612 (1997).

[13] A. Datta, Y.-R. Wu, and Y.L. Wang, Phys. Rev. B 63, 125407 (2001).

[14] S. Habenicht, K. P. Lieb, J. Koch, and A. D. Wieck, Phys. Rev. B 65, 115327 (2002).

[15] S. Ichim and M. J. Aziz, J. Vac. Sci. Technol. B 23, 1068 (2005).

[16] T. C. Kim et al., Phys. Rev. Lett. 92, 246104 (2004); J. Erlebacher, M. J. Aziz, E. Chason, M. B. Sinclair, and J. A. Floro, ibid. 82, 2330 (1999).

[17] C. Volkert, J. Appl. Phys. 70, 3521 (1991); M. L. Brongersma et al., Appl. Phys. Lett. 71, 1628 (1997); S. G. Mayr, Y. Ashkenazy, and R. S. Averback, Nucl. Instrum. Methods Phys. Res., Sect. B 212, 246 (2003); S. Klaumünzer, ibid. 215, 345 (2004).

[18] H. Trinkaus, J. Nucl. Mater. 223, 196 (1995); 246, 244 (1997).

[19] J. F. Ziegler and J. P. Biersack, SRIM (TRIM 90) simulation package (1995), available from www.srim.org.

[20] Transmission electron micrographs [T. K. Chini et al., Phys. Rev. B 67, 205403 (2003)] show clearly such thickness variations in the penetration layer.

[21] An ion with energy $E\left(4.8 \times 10^{-15} \mathrm{~J}\right)$ heats a cylinder of length $R(25 \mathrm{~nm})$, diameter $d(12.5 \mathrm{~nm})$, and volume $V=$ $\pi d^{2} R / 4\left(3.1 \times 10^{-24} \mathrm{~m}^{3}\right)$ by $\Delta T_{0}=E /(\rho V C)=950 \mathrm{~K}$; with $\rho=2200 \mathrm{~kg} \mathrm{~m}^{-3}$ and $C=750 \mathrm{~J} \mathrm{~kg}^{-1} \mathrm{~K}^{-1}$ [23].

[22] F.S. Sherman, Viscous Flow (McGraw-Hill, New York, 1990), p. 252.

[23] H. Scholze and M. Lakin, Glass: Nature, Structure and Properties (Springer, Berlin, 1991). 